\title{
Biphasic constant-current stimulator for whole-animal shocking of marine animals
}

\author{
ANDREW D. MCCLELLAN \\ Department of Biomedical Engineering, Case Western Reserve University, Cleveland, Ohio 44106
}

\begin{abstract}
A constant-current stimulator delivering biphasic pulses was designed and constructed for whole-animal shocking during aversive conditioning of Pleurobranchaea, a marine gastropod mollusk used for behavioral and neurophysiological research. The advantages of whole-animal stimulation are discussed and results are briefly described with regard to the factors involved in evaluating the procedure. The circuit design and electrode considerations are presented in detail. The basic stimulator design has potential for use as a general instrument for behavioral and physiological research.
\end{abstract}

The "whole-animal" stimulator described below was designed and built to aversively condition Pleurobranch$a e a$, a marine gastropod mollusk suitable for investigating the neural correlates of behavior and learning (see Eisenstein, 1967 for a general discussion on invertebrate learning studies). Animals were shocked in moderately small rectangular aquaria through large platinum electrodes placed at each end (Mpitsos, Collins, \& McClellan, Note 1).

\section{DESIGN CONSIDERATIONS}

Whole-animal shocking of marine animals poses problems because the surrounding highly conductive sea water shunts much of the stimulating current. Since such large currents must be passed to obtain the current density within the animal necessary for functional stimulation, consideration must be given to the electrodes and the type of stimulation.

Typical electrode materials used for pulsed (ac) stimulation are stainless steel or platinum (Schwan, 1968). Their areas should be as large as possible to reduce the electrode-electrolyte impedance (Geddes, Da Costa, \& Wise, 1971). Unfortunately, both the potential and impedance of the interface vary nonlinearly with the current density at the electrode surface (Geddes et al., 1971). Voltage stimulation is therefore undesirable, since the amount of current depends on the electrode characteristics and vice versa. Constantcurrent stimulation is preferred, and the pulses should be biphasic, since this results in zero average current flow and reduced corrosion (Brummer \& Turner, 1977).

Maintaining a constant current density within the animal presumably leads to consistent levels of stimulation. Two factors influence this current density. (1) Changes in sea water salinity due to the animal's

A. D. M. is supported by NIH (PHS) Grant GM-01090. This work was supported by NSF Grant BNS 76-81233 awarded to G. Mpitsos. excretions or secretions and changes in water level alter the amount of current shunted by the sea water (the $\mathrm{R}_{\mathrm{sw}}$ term). ${ }^{1}$ (Keeping the water level constant and flushing the aquarium tank periodically should reduce or eliminate this problem.) (2) Changes in the size, shape, or orientation of the animal influence its effective resistance (the $R_{a}$ and $A_{a}$ terms), ${ }^{1}$ thus altering the current density within the animal. Small, shallow aquaria and large electrodes tend to reduce variations in the stimulating field with animal movement. To be effective, the electrodes should span the animal along the axis where movement is minimal.

\section{CIRCUIT DESCRIPTION}

The stimulator supplies repetitive $(5-50 \mathrm{~Hz})$ largecurrent $(20-1,000 \mathrm{~mA}$, peak to peak) biphasic pulses $(5.100 \mathrm{msec})$. The polarity of the output can be reversed and monophasic pulses are also available. The amplitude and duration symmetries of the biphasic waveform are independently adjustable from $0 \%$ to $100 \%$. The stimulator can be easily converted to constant voltage output with high current passing capability by a single switch.

The logic circuitry (Figure 1) uses 555 integrated circuit timers (Hnatek, 1973) to control pulse timing parameters. A specially designed oscillator $\left(\mathbf{M}_{1}\right)$ determines the repetition rate by variation of $R_{1}$. The oscillator (McClellan, 1976) triggers a monostable $\left(M_{2}\right)$ which determines the total biphasic pulse duration by variation of $\mathbf{R}_{\mathbf{2}}$. The monostable acts as a ramp generator (Hnatek, 1973); its output $\left(\mathrm{V}_{\mathrm{b}}\right)$ is fed to a comparator $\left(A_{2}\right)$. The comparator and transistors $Q_{4} \cdot Q_{6}$ divide the total monostable pulse into two parts, representing the two successive halves of the biphasic waveform. This is accomplished by triggering the comparator when the ramp output reaches a particular fraction of its full swing determined by the duration symmetry control $\left(R_{3}\right)$. Therefore, $R_{3}$ allows the relative percentage that the first pulse $\left(\mathrm{V}_{\mathrm{c}}\right)$ and second pulse $\left(\mathrm{V}_{\mathrm{c}}{ }^{\prime}\right)$ contribute to the total biphasic pulse duration to be varied from $0 \%$ to 


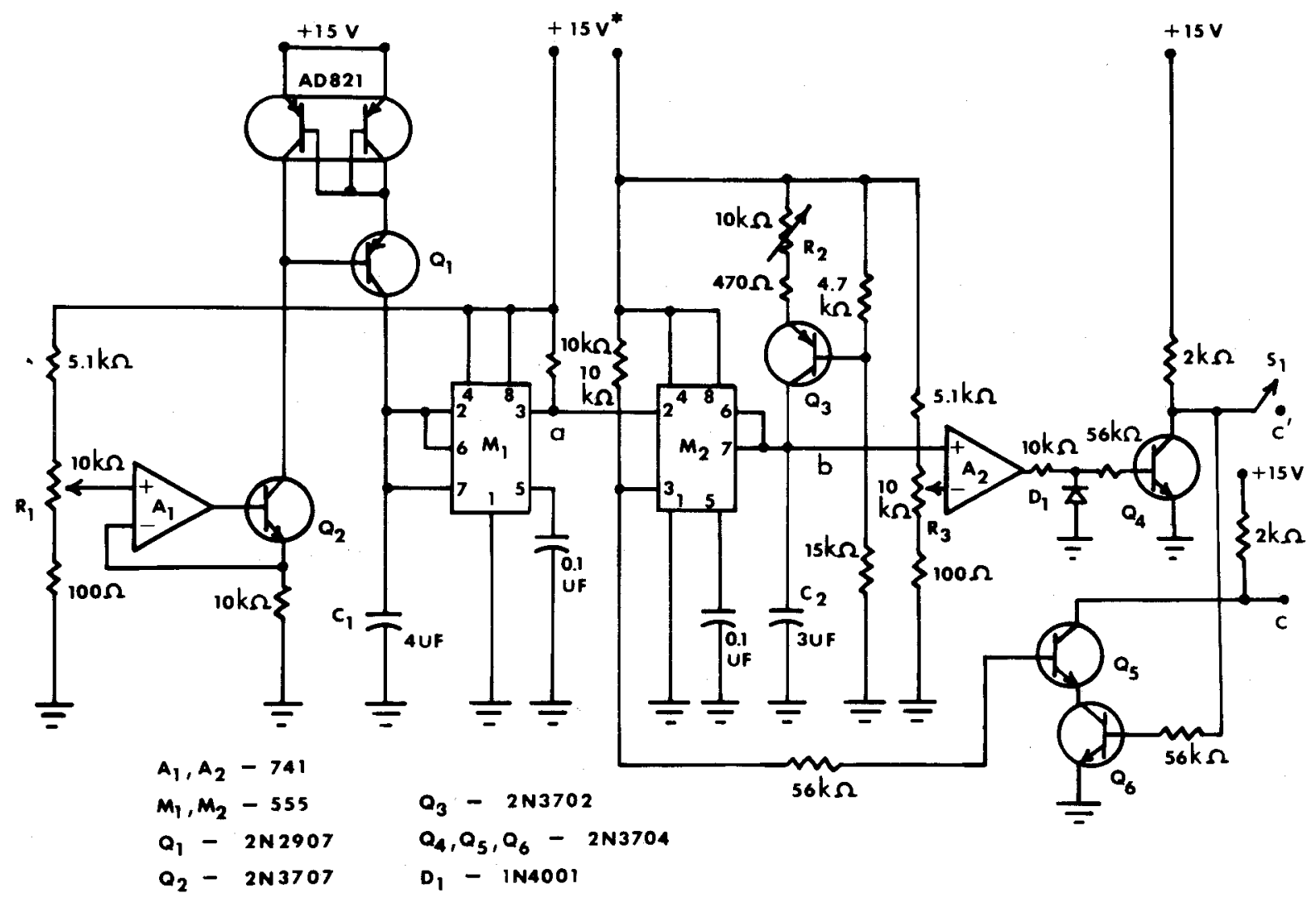

Figure 1. Timing circuitry for controlling the repetition rate and biphasic pulse duration characteristics. The power supply lead to the timers $\left(^{*}\right.$ ) should be decoupled through $100 \Omega$ and .1 $\mu \mathrm{F}$ to ground to prevent false triggering. A general-purpose diode should be in-series with the power supply leads to the amplifiers to protect against transients during turn-on.

$100 \%$. Switch $S_{1}$ can be opened if only monophasic pulses are desired.

The output circuitry (Figure 2) controls the currentpulse polarity and magnitude. Since the terminals for the positive $\left(\mathrm{V}_{\mathrm{d}}\right)$ and negative $\left(\mathrm{V}_{\mathrm{d}}\right)$ phases both accept the same pulse shape, the inputs $\left(\mathrm{V}_{\mathrm{c}}\right.$ and $\left.\mathrm{V}_{\mathrm{c}}{ }^{\prime}\right)$ may be switched by $S_{2}$ to produce positive-negative or negativepositive current pulses. The amplitude symmetry control $\left(R_{4}\right)$ allows the relative percentage that the positive $\left(V_{e}\right)$ and negative $\left(\mathrm{V}_{\mathrm{e}}{ }^{\prime}\right)$ phases contribute to the peak-to-peak current pulse to be varied from $0 \%$ to $100 \%$. The peakto-peak amplitude control $\left(R_{5}\right)$ produces a biphasic voltage pulse $\left(\mathrm{V}_{\mathrm{g}}\right)$ which is ready to be converted into a current pulse.

Amplifier $\mathbf{A}_{3}$ and the subsequent transistor circuitry form a precision voltage-to-current converter with a Darlington push-pull transistor pair (Millman \& Halkias, 1972) acting as a biphasic high-current source. With $S_{3}$ in the current mode position (I), the output current must pass through the current-sensing resistor $\left(R_{s}\right)$. The voltage developed across this resistor is a measure of the current. This voltage $\left(V_{i}\right)$ is fed back to $A_{3}$ as part of a high gain negative feedback control loop to adjust its output so that $V_{i}=V_{g}{ }^{2}$ Thus, the biphasic voltage
$\left(V_{g}\right)$, is directly related to the output current $\left(I_{o}=\right.$ $V_{i} / R_{s}=V_{g} / R_{s}$ ). An important factor of the output transistors is that they are operated class $C$ (normally OFF), with no current drain between pulses.

\section{APPLICATIONS}

The basic design, with minor component modifications in some cases, can be used as a general-purpose stimulator for both behavioral and physiological research. Localized or whole-animal stimulation of either aquatic or terrestrial animals is possible provided that the current level is appropriate.

It is important to remember that surprisingly small currents can cause fibrillation of the heart (Strong, 1970). Current flow is often sufficiently limited by body resistance when accidental contact is made with a voltage source; however, this is not the case with constant-current sources.

\section{EVALUATION}

Although whole-animal shocking has advantages, it is not always suitable. Localized shock applied to the 


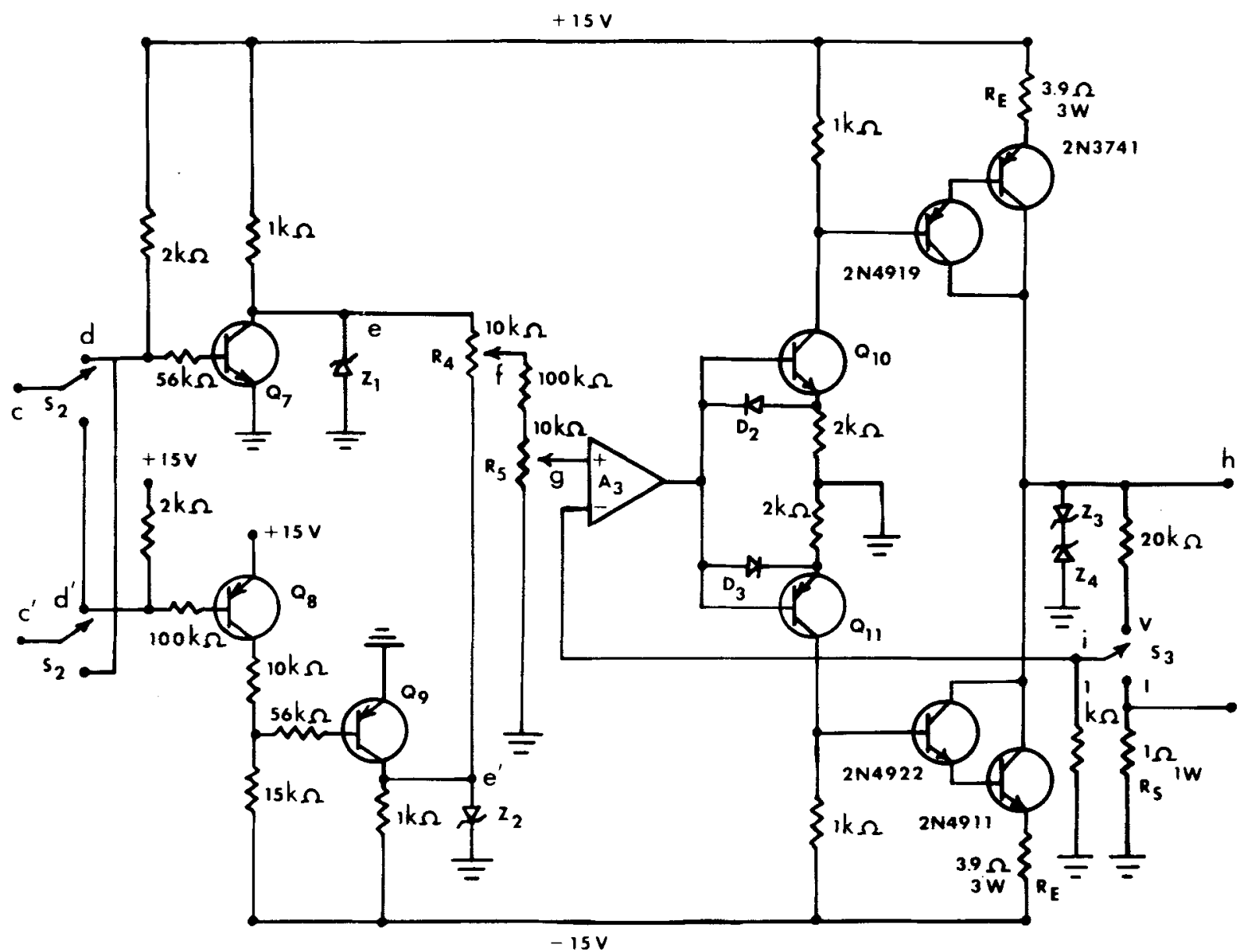

$$
\begin{aligned}
& A_{3}-741 D_{1}, D_{2}, D_{3}-1 N 4001 \\
& Q_{7}, O_{10}-2 N 3704 \\
& a_{8}, Q_{9}, O_{11}-2 N 3702 \\
& z_{1}, z_{2}-1 N 916 B \\
& z_{3}, z_{4}-I N 5349
\end{aligned}
$$

Figure 2. Amplitude control and output stage for delivering high current pulses. Switch $S_{3}$ allows either constant current mode (I, 20-1,000 mA, peak to peak) or constant voltage mode (V, 4-20 V, peak to peak). The power supply leads to $A_{3}$ should be protected against transients.

anterior part of Pleurobranchaea during aversive conditioning usually results in regional withdrawal from the general area of stimulation (i.e., avoidance response). Whole-animal stimulation (symmetrical 15-msec 400$\mathrm{mA}$ peak-to-peak biphasic current pulses at $15 \mathrm{~Hz}$ ) in small aquaria that prevent escape result in suppressed feeding responses and writhing movement over the animal's mid-dorsal surface; however, there is no localized withdrawal (Mpitsos, Collins, and McClellan, Note 1). Therefore, after the animals are conditioned, food suppresses the normally innate feeding response, but does not elicit withdrawal as it does in the localized shocking paradigm (Mpitsos \& Collins, 1975). This is to be expected since the conditioned response to food resembles the unconditioned response elicited by the particular type of stimulation used during conditioning.

Whole-animal stimulation does not provide a welldefined stimulus from which to withdraw, since the noxious stimulus, in effect, surrounds the animal. If withdrawal is the response of interest, whole-animal shocking is probably not appropriate. An alternative is the conditioning of escape responses in larger tanks, as is 
done with goldfish shuttle boxes. These considerations and perhaps others should be evaluated for individual conditioning paradigms.

\section{REFERENCE NOTE}

1. Mpitsos, G., Collins, S., \& McClellan, A. D. Learning: $A$ model system for physiological studies. Submitted for publication.

\section{REFERENCES}

Brummer. S. B. \& \& TURNER, M. J. Electrochemical considerations for safe electrical stimulation of the nervous system with platinum electrodes. IEEE Transactions in Bio-Medical Engineering, 1977, BME-24, 59-63.

EISENSTEIN, E. The use of invertebrate systems for the bases of learning and memory. In G. Quarton, T. Melnechuk, \& F. O. Schmitt (Eds.), The neurosciences: $A$ study program. New York: Rockefeller University Press, 1967. Pp. 653-664.

Geddes, L.. DA Costa, C.. \& Wise, G. The impedance of stain. less steel electrodes. Medical Biological Engineering. 1971, 9. $511-521$.

HNATEK. E. Put the IC timer to work in a myriad of ways. EDN, 1973, 18, 54-58.

MCClellan, A. Current source and 555 timer make linear v-to-f converter. Electronics, 1976, 49. 108-109.

Millman. J., \& Halkias. C. Integrated electronics: Analog and digital circuits and systems. New York: McGraw-Hill, 1972, Pp. 695-696.
Mpitsos, G.. \& Collins. S. Learning: Rapid aversive conditioning in the gastropod mollusk Pleurobranchaea. Science, 1975, 188, 954-957.

SCHWAN, H. Electrode polarization impedance and measurements in biological materials. Annals of the New York Academy of Sciences, 1968, 148, 191-209.

Strung, P. Biophysical Measurements. Beaverton: Tektronix, Inc., 1970. Pp. 255-259.

\section{NOTES}

1. A very simplified expression for the current density within the animal is formulated by assuming that the effective resistance of the animal is in parallel with the resistance of the shunting sea water. Thus,

$$
\mathbf{J}_{\mathbf{a}}=\frac{\mathbf{R}_{\mathbf{s w}}}{\mathbf{R}_{\mathbf{s} \mathbf{w}}+\mathbf{R}_{\mathbf{a}}} \cdot \frac{\mathbf{I}}{\mathbf{A}_{\mathbf{a}}}
$$

where $\mathbf{J}_{\mathbf{a}}=$ current density within the animal, $\mathbf{I}=$ total current output of the stimulator, $\mathbf{A}_{\mathbf{a}}=$ effective cross-sectional area of the animal perpendicular to the current flow, $\mathbf{R}_{\mathrm{sw}}=$ effective resistance of the sea water, and $R_{a}=$ effective resistance of the animal, which partially depends on $A_{a}$.

2. If functional stimulation levels are highly variable (see Design Considerations), the current flowing through the animal may have to be monitored and fed back, instead of $V_{1}$, to control the output current level.

(Received for publication July 10, 1977; revision accepted September $7,1977$. 